\title{
Sentinel Lymph Node Biopsy Followed by Axillary Lymph Node Biopsy
}

National Cancer Institute

\section{Source}

National Cancer Institute. Sentinel Lymph Node Biopsy Followed by Axillary Lymph Node Biopsy. NCI Thesaurus. Code C120282.

A staging procedure consisting of sentinel node biopsy followed by surgical evaluation of the axillary lymph nodes. 